\title{
SIMULATED IN-HOME TELETREATMENT FOR ANOMIA
}

\author{
LAMBERT DECHÊnE ${ }^{1}$, M.O.A., MiChEL TOUSIGNANT ${ }^{1}$, PT., PH.D., PATRICK \\ BOISSY ${ }^{1}$, PH.D., JOËL MACOIR ${ }^{2}$, PH.D., SERGE HÉROUX ${ }^{3}$, M.SC., MATHIEU \\ HAMEL ${ }^{1}$, ING., M.SC.A., SiMON BRIÈrE ${ }^{1}$, ING., M.SC.A., CATHERINE PAGÉ ${ }^{1}$, M.SC. \\ ${ }^{1}$ Research Centre on Aging, Sherbrooke Geriatric University Institute, FaCUlty of Medicine

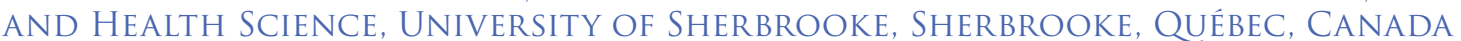 \\ ${ }^{2}$ LAVAl UNIVERSITY, FACUlTy OF MEDICINE, QUÉBEC, QUÉBEC, CANADA \\ ${ }^{3}$ CSSS du laC-Des-DeuX-MOnTagnes, Saint-Eustache, QuÉBeC, Canada
}

\section{ABSTRACT}

This pilot study explored the feasibility of in-home teletreatment for patients with post-stroke anomia. Three participants over 65 years of age suffering from post-stroke anomia were treated in this pre/post-intervention case study. They received 12 speech therapy teletreatments (two sessions/week for 6 weeks) aimed at improving confrontation naming skills. Half of the failed items from a set of 120 preselected stimuli were trained during treatment (Block A-trained stimuli) while the other half served as controls (Block B-untrained stimuli). Variables measured were: 1) efficacy of treatment (performance on Block-A vs. Block B Stimuli), and 2) participants' satisfaction with teletreatment (using a French adaptation of the Telemedicine satisfaction questionnaire). All participants showed a clinically relevant improvement on confrontation naming of trained items and less improvement for untrained items. The researchers also obtained high satisfaction scores on the questionnaire (above 57/60). This pilot study supports the feasibility of speech therapy teletreatments applied to neurological language disorders.

Keywords: Anomia, aphasia, speech therapy, stroke, telepractice, teletreatment

\section{INTRODUCTION}

The incidence of stroke is increasing in industrialized countries, particularly among seniors. After 55 years of age, the risk of stroke doubles every 10 years (Appel \& Llinas, 2005). After motor problems, language function impairment (aphasia) is one of the most common and devastating cognitive deficits caused by stroke lesions (Albert, 1998; Fridriksson, 2010). It is estimated that one-third of people with vascular cerebral lesions suffer from aphasia (Pedersen, Vinter, \& Olsen, 2004), which is a multimodal disorder affecting auditory comprehension, reading, oral-expressive language and writing (Mazeaux, Brun, \& Pélissier, 2000; McNeil \& Pratt, 2001). One of the most common manifestations of aphasia is anomia, defined as a problem in word finding. Anomia alters conversational language by inducing delays, blanks and word selection errors into one's verbal output. It often has a significant impact on the individual's family and social life. Anomia can be measured with specific assessment tasks (e.g., confrontation naming, verbal fluency).

The prognosis for aphasia depends on various factors, such as the initial severity of language impairments, the type of aphasia, and the impact of speech therapy (Kertesz \& McCabe, 1977; Pedersen, et al., 2004; Robey,
1998). Treatment usually begins in acute care and continues when the patient is transferred to an intensive functional rehabilitation unit. After discharge, the motor disorders and fatigue associated with stroke are often obstacles that hinder traveling to a clinic for speech therapy. This difficulty is reflected in the high demand for home care services. In the province of Quebec, Canada, in-home follow-up in speech therapy is almost inexistent (Québec, 2003), which compromises patients' recovery. In rural areas, travel distances and access to specialized services are also issues that can compromise language interventions (Wilson, 2002). Thus, alternatives to existing rehabilitation models must be pursued in order to increase access to rehabilitation services and meet the current needs of the population (Ministère de la santé et des services sociaux, 2003, 2004).

In-home speech therapy teletreatment, a telehealth application that uses telecommunications to deliver speech therapy rehabilitation services, is growing as a complementary or alternative approach in the rehabilitation field. It has been identified as a promising alternative that can improve access to health care in general (Romanow, 2002). The rationale for in-home teletreatment is to expand and facilitate the delivery of speech therapy services to people who cannot access 
them due to unavailability of specialists, language barriers, travel distances and costs, weather conditions, or impaired mobility (Canadian Association of SpeechLanguage Pathologists and Audiologists, 2005; American Speech-Language-Hearing Association, 2006).

Various published studies have demonstrated acceptable reliability between teleconsultation and conventional (in person) consultation for the assessment of neurological language (Brennan, Georgeadis, Baron \& Barker, 2004; Georgeadis, Brennan, Barker \& Baron, 2004; Hill, Theodoros, Russel \& Ward, 2009; Theodoros, Hill, Russell, Ward \& Wootton, 2008) and speech disorders (Constantinescu et al., 2010; Hill, Theodoros, Russell \& Ward, 2009; Theodoros, Russell, Hill, Cahill \& Clark, 2003). However, in this particular domain, articles on teletreatment are scarce. They were published in the last year or so and exclusively addressed treatment of motor speech disorders (e.g. dysarthria). Of these studies (Beijer, Rietveld, Hoskam, Geurts \& de Swart, 2010; Constantinescu et al., 2011; Constantinescu et al., 2010; Lasker, 2010), only two involved home-based teletreatment between patient and therapist.

One study demonstrated the effectiveness of a specific apraxia of speech treatment protocol (the Motor Learning Guided approach) delivered by Skype and in person sessions, combined with daily independent practice with a speech generating device (Lasker, 2010). Treatment in both conditions resulted in similar outcomes in terms of intelligibility, immediacy of response, and naturalness of speech. However, in these results, the contribution of daily independent practice with a speech model is unclear. Also, because this case study involved a 28-year-old patient familiar with modern technology, it is difficult to generalize the applicability of this treatment modality to an elderly population.

The other study (Constantinescu, et al., 2010) investigated the validity and feasibility of online delivery of the Lee Silverman Voice Treatment (LSVT), an intensive speech treatment for Parkinson's disease. The patient was a 65-year-old living $90 \mathrm{~km}$ from the speech and language pathology clinic. A PC-based videoconferencing system was used, operating at $128 \mathrm{kbit} / \mathrm{s}$ over the public telecommunications network. After treatment was completed, the patient showed substantial improvements on different acoustic and perceptual variables (vocal sound pressure levels, vocal duration, voice quality and overall speech intelligibility) similar in degree to treatment outcomes reported in the LSVT literature. Interestingly, this participant reported a preference for online sessions for the management of his speech disability because they saved time and meant he did not have to travel.

The results are encouraging, yet the feasibility, efficacy and adaptability of this novel service delivery method needs to be documented with elderly people experiencing neurological language disorders. The multimodal nature of aphasia and its receptive counterparts may be more challenging to address remotely. In this context, the main objective of our study was to explore the feasibility of inhome teletreatment designed for patients with post-stroke lexical problems.

\section{METHODS}

\section{DESIGN}

The design used in this feasibility study is a pilot study with a pre/post-interve ntion measurements without a control group.

\section{SAMPLE}

In this pilot study, the target population was individuals at least 65 years of age with post-stroke aphasia. Participants were recruited at the Health and Social Services Center, University Institute of Geriatrics of Sherbrooke (CSSS-IUGS). One of the objectives of this health care facility is to provide functional intensive rehabilitation services to older people after discharge from an acute care hospital.

Inclusion criteria for participants were that they: 1) experience oral expression difficulties related to a stroke, 2) exhibit an oral receptive level sufficient to participate in remote treatment (score $>33$ on the oral comprehension subtest of the Montreal-Toulouse Linguistic Examination of Aphasia Protocol) (Nespoulous et al., 1992), and 3) demonstrate minimum skills in implementing oral tasks (score $>17$ on the repetition and reading subtests of the Montreal-Toulouse Linguistic Examination of Aphasia Protocol) (Nespoulous, et al., 1992).

Patients were recruited following discharge from the intensive functional rehabilitation unit or the day hospital. They were screened by a speech therapist to verify their eligibility based on medical records. When a potential participant was identified, the speech therapist presented the project and verified the person's interest in participating. If the patient agreed, he/she was invited to sign a consent form, which was approved by the institution's ethics board.

\section{TECHNOLOGICAL INFRASTRUCTURE FOR TELEREHABILITATION}

Based on experience from two previous studies (Boissy, Tousignant \& Corriveau, 2005; Tousignant, Boissy, Corriveau \& Moffet, 2007), a speech therapy teletreatment platform was developed and refined. The platform included various components designed to create a user-friendly experience for both the clinician, and the patient at home. Although similar in many ways, two different systems were used to provide speech therapy 
services: an "in-home" system and a clinician system. The telehealth platform and software interface for both systems are illustrated in Figure 1.

The core of these systems is the videoconferencing system (Tandberg 550 MXP), which uses a h.264 video codec, and integrates a pan-tilt-zoom (PTZ) wide angle camera and an omnidirectional microphone. The system is mounted over a 20-inch LCD screen, which displays the video received from the other end. Audio can be played using external speakers placed on both sides of the screen. A touch screen tablet PC was added to the "in home" system to display pictures used in therapy, allow interaction with them, and enable participants to write directly on screen.

Sessions were established using a high speed Internet connection over cable or DSL that was installed into the patient's home. The videoconferencing system and streamed pictures that were displayed on the tablet PC used no more than $600 \mathrm{kbps}$.

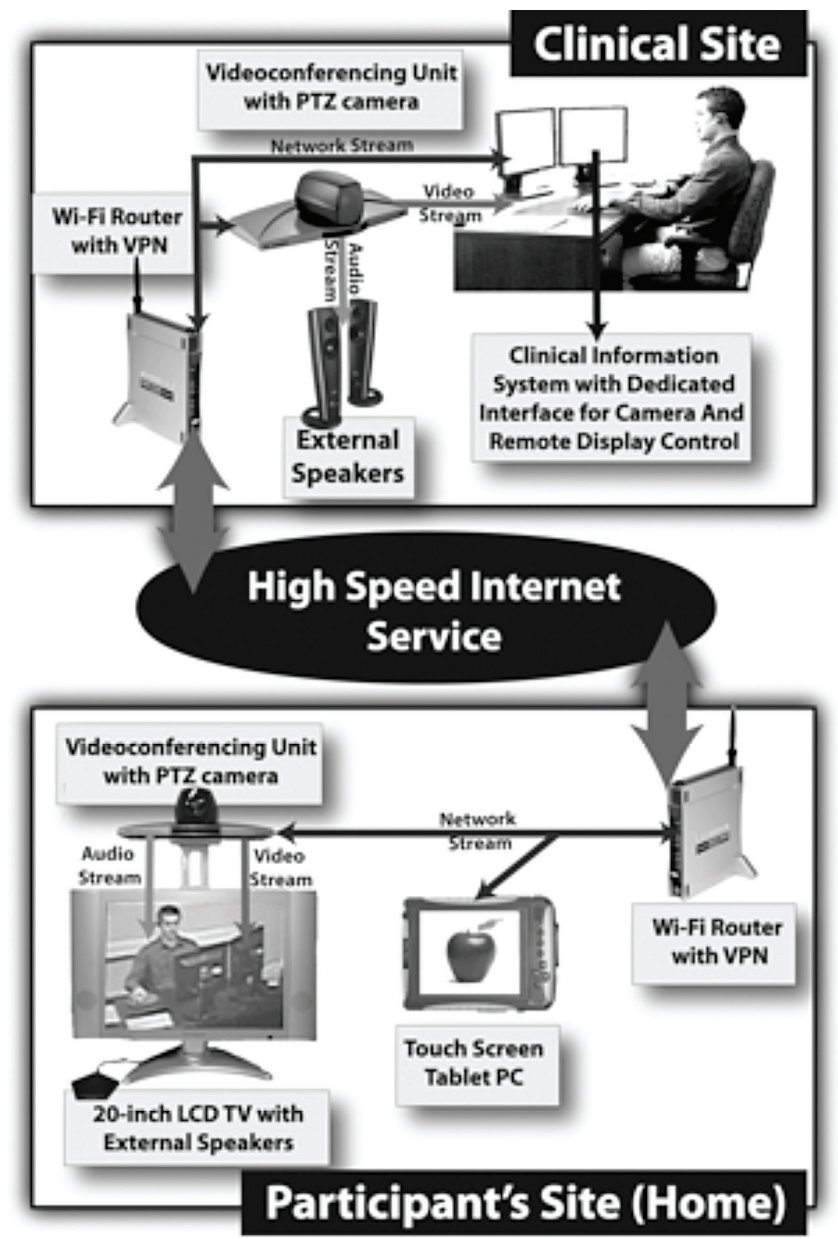

Figure 1: Technological infrastructure for speech therapy teletreatment services.

\section{SPEECH THERAPY TELETREATMENT}

A lexical approach was chosen for the treatment of anomia. Various authors have demonstrated the efficacy of transposition tasks (repetition, oral reading, copy, spelling to dictation) and cuing (orthographic and phonemic) to heighten access to word form (Basso, Marangolo, Piras \& Galluzzi, 2001; Best, 2002). However, these types of approaches are reported to be item-specific (i.e., the treatment effects are limited to stimuli practiced during training with no generalization effects).

So as to tailor the intervention to each patient's level and needs, a confrontation naming test was designed using a published and thoroughly studied set of visual stimuli (Snodgrass \& Vanderwart, 1980). A total of 120 simple line drawings were chosen, representing various semantic categories, on the basis of unequivocal responses to the stimuli and lexical frequency ( 30 high, 45 moderate and 45 low frequency words). They were presented to the patients in two baseline assessments, and success (defined here as a correct answer obtained within 5 seconds) or failure was recorded for each of them. Pairs of failed items were then created according to lexical frequency and animate/ inanimate distinctions. Within each pair, patients chose one item for training on the basis of their preferences (Block A-trained stimuli) while the others served as the control stimuli (Block B-untrained stimuli).

Each treatment session was composed of the following: 1) a pre-assessment of trained stimuli (Block $A$ ) to establish the pre-treatment baseline, 2) three different lexical tasks involving the stimuli, 3) specific circumlocutions training (added for its ecological pertinence but not assessed), and 4) a post-assessment of trained stimuli (Block A) to establish the effect of the treatment. Two trained speech therapists were involved in these treatments. Each participant received a total of 12 teletreatments (two onehour treatments per week for six weeks) delivered by the same speech therapist. The training tasks performed, which all put emphasis on the word form, are listed in Table 1.

Table 1: Training tasks performed by participants

- Delayed repetition

- Delayed oral reading

- Oral reading + delayed copy

- Spelling to dictation

- Oral confrontation naming with orthographic cuing (first or last syllable)

- Written confrontation naming with orthographic cuing (first grapheme or syllable)

- Word to picture matching (visual distractors) + oral reading

- Rhyme to picture matching

- Syllabic fusion

- Picture and word sequencing

- Closing tasks (evoking a word with the first or last written or oral syllable)

- Word identification with spelling

- First letter or syllable identification 
Considering the exploratory nature of the study (pilot study) and the novelty of the platform used, the teletreatments for the first participant were delivered at the CSSS-IUGS. The participant sat in a room next to the speech therapist's office so that they could interact quickly and resolve technological problems more easily. In order to simulate the home environment, the connection between the two rooms was a simulation of a residential network. After some adjustments were made, the two other participants received their teletreatments at home, where they lived independently; they were connected via a high speed residential Internet network to the speech therapist's office.

In this pilot study, the two participants at home were accompanied by a speech therapist during the first two sessions so they could learn the procedures for connecting, manipulating the touch screen tablet PC, and disconnecting. These procedures were kept to a minimum with a single switch button activating the system and all computer exercises controlled remotely.

\section{DEPENDENT VARIABLES}

\section{EFFICACY OF SPEECH THERAPY}

To evaluate the efficacy of treatment, pre/postassessments were done using the confrontation naming test designed for this study. The number of successfully trained stimuli (Block A) was compared to the status of a comparable set of untrained stimuli (Block B). According to the hypothesis, there should be an improvement on the trained stimuli after the intervention but not on the control stimuli.

\section{SATISFACTION OF PARTICIPANTS}

Satisfaction of the participants with the teletreatment was assessed using a French adaptation of the Telemedicine Satisfaction Questionnaire (Yip, Chang, Chan \& MacKenzie, 2003). This questionnaire contains 12 items scored on a five-point Likert scale, where 1 represents the lowest and 5 the highest level of satisfaction (total score: 60 ). Quality of communication between patient and therapist, accessibility and needs that were met are the domains assessed. Semi-structured interviews were also conducted to gather participants' impressions of their experience.

\section{DATA COLLECTION PROCEDURES}

Data were collected by an independent speech therapist. Trained and control stimuli were selected according to the results of two baseline assessments on the confrontation naming test, with the T2 measurement one week after the T1 measurement. At T1, selected subtests of the Montreal-Toulouse Linguistic Examination of Aphasia Protocol were also administered to satisfy the inclusion criteria. Thereafter, participants received 6 weeks of treatment. One week after the treatment period, a T3 measurement using the confrontation naming test assessed clinical improvement. The satisfaction questionnaire and semi-structured interviews were also completed at that time.

Figure 2: Assessment times

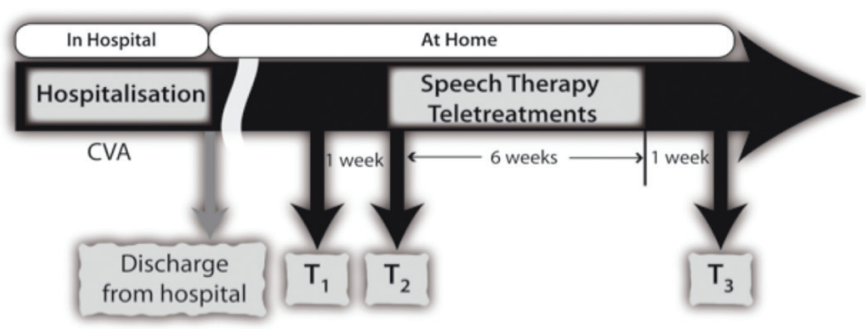




\section{RESULTS}

\section{DESCRIPTION OF THE SAMPLE}

Three French-speaking patients who suffered a stroke (cerebrovascular accident: CVA) with language disorders were recruited. Characteristics of the sample are described in Table 2.

Table 2: Characteristics of the participants

\begin{tabular}{|c|c|c|c|}
\hline Characteristics & Participant 1 & Participant 2 & Participant 3 \\
\hline Sex & Man & Woman & Woman \\
\hline Age (years) & 80 & 79 & 71 \\
\hline Years of schooling (years) & 18 (university) & 10 & 16 (university) \\
\hline Lesion site & $\begin{array}{l}\text { Left temporal } \\
\text { haematoma }\end{array}$ & $\begin{array}{l}\text { Ischemia in the } \\
\text { area of the middle } \\
\text { cerebral artery } \\
\text { with extensive } \\
\text { thalamic and } \\
\text { cortical damage }\end{array}$ & $\begin{array}{l}\text { Alexia without } \\
\text { agraphia }\end{array}$ \\
\hline Aphasia type & $\begin{array}{l}\text { Fluent aphasia with } \\
\text { anomia }\end{array}$ & Broca's aphasia & $\begin{array}{l}\text { Fluent aphasia } \\
\text { with anomia }\end{array}$ \\
\hline Post-CVA delay (months) & 2 & 8 & 6 \\
\hline $\begin{array}{l}\text { Having regular speech } \\
\text { therapy during the project? }\end{array}$ & Yes & No & Yes \\
\hline Familiar with computer? & No & Yes & No \\
\hline
\end{tabular}

Participants 1 and 3 were having regular speech therapy at the time of the experimental intervention (one session/week), but the goals and materials used during those sessions were unrelated to the anomia teletreatment : calculus, reading skills (decoding at the syllable level), and comprehension of paragraphs.

\section{EFFICACY OF THE TELETREATMENT}

Clinical outcomes improved for all three participants. After 6 weeks of teletreatment, they showed a clinically relevant improvement on confrontation naming of the trained items (Block A). As expected, there was less improvement for the untrained stimuli (Block B). Note that the number of trained and control stimuli varied from one participant to the next according to their initial performance on the confrontation naming test and the possibility of matching stimuli (19, 15 and 20 stimuli in each block, respectively).
Figure 3: Confrontation naming (Block $\mathrm{A}$ and Block $\mathrm{B}$ ) at $\mathrm{T} 1, \mathrm{~T} 2$ and $\mathrm{T} 3$
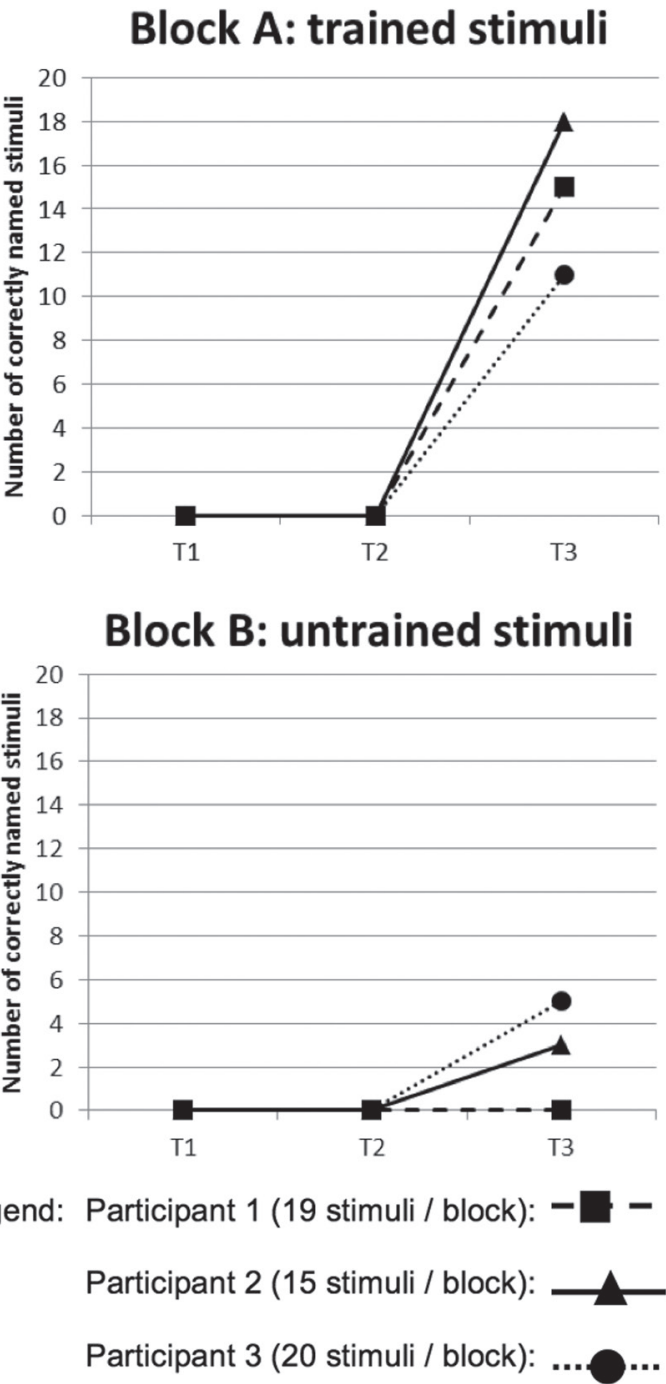

The improvement on trained items as measured throughout the treatment sessions followed almost the same pattern for participants 1 and 3 . The number of succeeded (correctly named) stimuli gradually increased to reach an almost perfect score at the end of the treatment period, with a slight decline in performance at T3. Participant 2 had a slightly different evolution profile. A peak performance was obtained sooner, halfway through the treatment process, with lower performances during the last few sessions, comparable to the results obtained at T3. 
Figure 4: Confrontation naming (Block $A$ ) throughout the treatment period

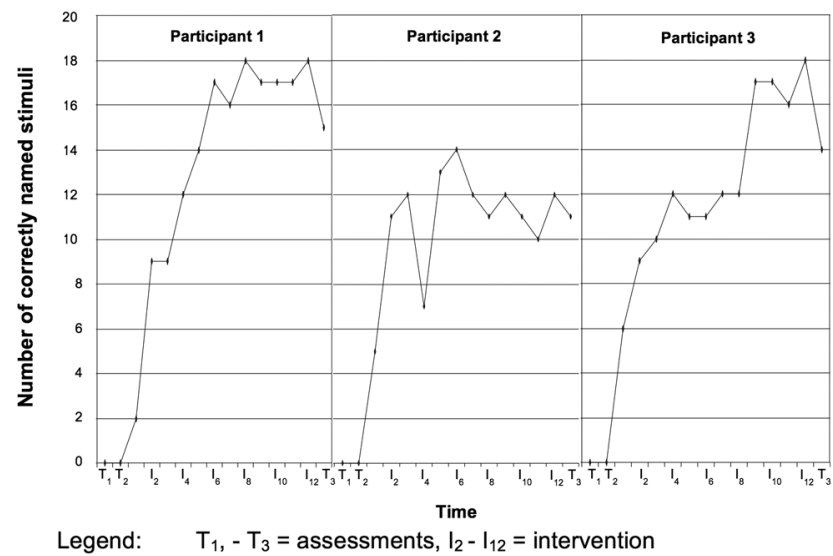

\section{SATISFACTION OF PARTICIPANTS}

Teletreatment was considered highly satisfactory for every patient. Their scores on the satisfaction questionnaire were all above 56/60 (56/60 for participant $1,58 / 100$ for participant 2, 58/60 for participant 3). Examples of comments from the interviews confirmed their satisfaction with their experience:

- "It was easy [using the technology]. He tells us what to do. No, I didn't find it difficult really. Actually, it was fun!" (participant 1)

- "It's the same as if you were in front of me! (...) Speech therapy sessions are now over, and I can't believe how it helped me! Now I'm able to do things I couldn't do before. If I have something to write, I will just write it down!" (participant 2)

- "I didn't hate it! I think it was adequate [teletreatments]. There weren't a lot of problems [with the Internet connection]. A few times we lost it for a few minutes, but it really gave us the advantage of less traveling." (participant 3)

\section{DISCUSSION}

The main objective of this pilot study was to explore the feasibility of in-home teletreatment designed for patients with post-stroke anomia by assessing its efficacy and evaluating participants' satisfaction with this novel service delivery method. To our knowledge, this is the first study to investigate teletreatments from a service center to a patient's home in the field of neurological language disorders.

Overall, our study gave a first indication of its clinical relevance. According to the results, teletreatment appears to be an innovative and effective way to provide anomia rehabilitation post-stroke. The pre/post-intervention changes observed on the confrontation naming task, as well as the improvement on the trained stimuli during treatment, are good indicators of the efficacy of this therapy.

The evolution of each participant's naming abilities showed a similar pattern: there was a rapid improvement on the trained stimuli, reaching an almost perfect score $(100 \%)$ at some point during the intervention process. However, for participant 2, the results obtained on the last few sessions showed a slight decline in performance. This could be explained by the health status and corresponding state of mind of that patient, who had to visit the hospital's emergency room during that period. For participants 1 and 3 , there was a slight decline on the trained stimuli when tested one week post-treatment (T3). This decline in performance could be due to increased fatigue during a lengthier assessment task (having to name 120 items instead of 20 or less) or a lack of maintenance of the treatment effects. The design of this study did not allow us to determine this with any certainty. In the future, a longer follow-up could examine acquisition maintenance after treatment.

The high scores on the satisfaction questionnaire showed that these elderly patients appeared to accept this new service delivery method, even more than therapists, according to the results of other studies (Dunkley, Pattie, Wilson \& McAllister, 2010; Schopp, Johnstone \& Merveille, 2000). The adjustments made to the technology from one participant to the next resulted in more user-friendly equipment and simplified the connection process. After minimal training, all participants were able to function independently with the technology. In addition, they mentioned that the platform was easy to use even though the majority did not have any knowledge about computers. 


\section{LIMITATIONS}

Certain biases may have affected the internal validity of the study and warrant discussion. The participants were not randomly recruited. It is possible that the participants included in the study were more enthusiastic about modern technology and agreed more readily to participate. This could lead to a satisfaction bias.

No comparison group was included in the study, which limits the extent of the results, even though performances on the trained and control stimuli were compared.

Moreover, the number of baseline measurements (T1 and T2) was insufficient to make sure that these stimuli could not be named by the participants prior to treatment.

Information bias may also be an issue. Despite the standardized outcome measures used, assessors during the treatment phase were not independent of the intervention because of the limited availability of speech therapists.

Concerning the telerehabilitation platform used in the study, observations made by the therapists and participants indicate that there is still room for improvement. First, the use of two screens at the patient's home was not ideal (one for videoconferencing and the other to present visual stimuli and receive answers from patients). Indeed, this affected the participants' concentration. The size of the screen used to present the visual cues should be enlarged, given the visual problems often associated with age and stroke. The distance of the camera from the therapist's screen is also an issue that may prevent eye contact with the patient, as mentioned in another study (Constantinescu, et al., 2011). Finally, audio delays encountered during some sessions affected turn-taking in conversation, which could have a significant impact when working with patients with logorrhea.

\section{CONCLUSION}

This pilot study proved the feasibility of teletreatment applied to neurological language disorders as a complement or alternative to in-person interventions. The preliminary results justify conducting more in-depth research on teletreatment with this population to address the weaknesses of the present study, including a control group and a bigger sample size to assure external validity.

\section{REFERENCES}

1. Albert, M. (1998). Treatment of aphasia. Archives of Neurology, 55, 1417-1419.

2. Appel, L., \& Llinas, R. (2005). Hypertension and stroke. The Johns Hopkins White Papers ed., Baltimore, Maryland: Johns Hopkins Medicine.

3. American Speech-Language-Hearing Association. (2005). Speech-language pathologists providing clinical services via Telepractice: Position statement. Retrieved from www.asha.org/docs/html/PS2005-00116.html

4. Basso, A., Marangolo, P., Piras, F., \& Galluzzi, C. (2001). Acquisition of new "words" in normal subjects: a suggestion for the treatment of anomia. Brain and Language, 77(1), 45-59.

5. Beijer, L. J., Rietveld, T. C., Hoskam, V., Geurts, A. C., \& de Swart, B. J. (2010). Evaluating the feasibility and the potential efficacy of e-learning-based speech therapy (EST) as a web application for speech training in dysarthric patients with Parkinson's disease: A case study. Telemedicine Journal and E-Health, 16, 732-738.

6. Best, W., Herbert, R., Hickin, J., Howard, D., \& Osborne, F. (2002). Phonological and orthographic facilitation of word retrieval in aphasia: short and long term effects. Aphasiology, 16, 151-168.

7. Boissy, P., Tousignant, M., \& Corriveau, H. (2005) Téléréadaptation à domicile pour le suivi des personnes ayant subi une arthroplastie du genou : Étude étude pilote. Disability and Rehabilitation: Assistive Technology, 1, 209-216.

8. Brennan, D. M., Georgeadis, A. C., Baron, C. R., \& Barker, L. M. (2004). The effect of videoconference-based telerehabilitation on story retelling performance by braininjured subjects and its implications for remote speechlanguage therapy. Telemedicine Journal and E-Health, 10, 147-154.

9. Canadian Association of Speech-Language Pathologists and Audiologists. (2006). Position paper on the use of telepractice for CASLPA speech-language pathologists and audiologists. Retrieved from http://www.caslpa.ca/ PDF/position\%20papers/telepractice.pdf

10. Constantinescu, G., Theodoros, D., Russell, T., Ward, E., Wilson, S., \& Wootton, R. (2010). Assessing disordered speech and voice in Parkinson's disease: A telerehabilitation application. International Journal of Language \& Communication Disorders, 45,630-644.

11. Constantinescu, G. A., Theodoros, D. G., Russell, T. G., Ward, E. C., Wilson, S. J., \& Wootton, R. (2010). Homebased speech treatment for Parkinson's disease delivered remotely: A case report. Journal of Telemedicine and Telecare, 16, 100-104

12. Constantinescu, G., Theodoros, D., Russell, T., Ward, E., Wilson, S., \& Wootton, R. (2011). Treating disordered speech and voice in Parkinson's disease online: a randomized controlled non-inferiority trial. International Journal of Language \& Communication Disorders, 46(1), 1-16.

13. Dunkley, C., Pattie, L., Wilson, L., \& McAllister, L. (2010). A comparison of rural speech-language pathologists' and residents' access to and attitudes towards the use of technology for speech-language pathology service delivery. International Journal of Speech-Language Pathology, 12, 333-343. 
14. Fridriksson, J. (2010). Preservation and modulation of specific left hemisphere regions is vital for treated recovery from anomia in stroke. Journal of Neuroscience, 30, 11558-11564.

15. Georgeadis, A. C., Brennan, D. M., Barker, L. M., \& Baron, C. R. (2004). Telerehabilitation and its effect on story retelling by adults with neurogenic communication disorders. Aphasiology, 18, 639-652.

16. Hill, A. J., Theodoros, D., Russel, T., \& Ward, E. (2009). Using telerehabilitation to assess apraxia of speech in adults. International Journal of Language \& Communication Disorders, 44, 731-747.

17. Hill, A. J., Theodoros, D., Russell, T., \& Ward, E. (2009). Using telerehabilitation to assess apraxia of speech in adults. International Journal of Language \& Communication Disorders, 44, 731-747.

18. Kertesz, A. \& McCabe P. (1977). Recovery patterns and prognosis in aphasia. Brain and Language, 100(1),1-18.

19. Lasker, J., Stierwalt, AGJ., Spence, M., \& Calvin-Root, C. (2010). Using webcam interactive technology to implement treatment for severe apraxia: A case example. Journal of Medical Speech -Language Pathology, 18, 71-75.

20. Mazeaux, J. M., Brun, V., \& Pélissier, J. (2000). Aphasie 2000- Rééducation et réadaptation des aphasies vasculaires. Paris: Massion.

21. McNeil, M., \& Pratt, S. (2001). Defining aphasia: Some theoretical and clinical implications of operating from a formal definition. Aphasiology, 15, 900-911.

22. Ministère de la santé et des services sociaux du Québec. (2003). Chez soi : Le premier choix. La politique de soutien à domicile. Ministère de la Santé et des Services sociaux du Québec: La Direction des communications, MSSS-Québec.

23. Ministère de la santé et des services sociaux du Québec. (2004). Chez soi : Le premier choix. Précisions pour favoriser l'implantation de la politique de soutien à domicile. Ministère de la Santé et des Services Sociaux, Québec: La Direction des communications, MSSSQuébec.

24. Nespoulous, J. L., Lecours, A. R., Lafond, D., Lemay, A., Puel, M., Joanette, Y., et al. (1992). Protocole MontréalToulouse d'examen linguistique de l'aphasie MT 86 module standard initial: Édition révisée. Isbergues.

25. Pedersen, P., Vinter, K., \& Olsen, T. (2004). Aphasia after stroke: Type, severity and prognosis. The Copenhagen Aphasia Study. Cerebrovascular Diseases, 17(1), 35-43.

26. Robey, R. R. (1998). A meta-analysis of clinical outcomes in the treatment of aphasia. Journal of Speech, Language, and Hearing Research, 41, 172-187.

27. Romanow, R. (2002). Guidé par nos valeurs: L'Avenir des soins de santé au Canada. Retrieved from www.hc.sc. gc.ca/français/soins/romanow/hcc0086.html

28. Schopp, L. H., Johnstone, B. R., \& Merveille, O. C. (2000). Multidimensional telecare strategies for rural residents with brain injury. Journal of Telemedicine and Telecare, 6(Suppl 1), S146-S149.

29. Snodgrass, J. G., \& Vanderwart, M. (1980). A standardized set of 260 pictures: Norms for name agreement, image agreement, familiarity, and visual complexity. Journal of Experimental Psychology: Learning, Memory, and Cognition, 6, 174-215.

30. Theodoros, D., Hill, A., Russell, T., Ward, E., \& Wootton, R. (2008). Assessing acquired language disorders in adults via the Internet. Telemedicine Journal of Electronic Health, 14, 552-559.
31. Theodoros, D., Russell, T. G., Hill, A., Cahill, L., \& Clark, K. (2003). Assessment of motor speech disorders online: A pilot study. Journal of Telemedicine and Telecare, 9 (Suppl 2), S66-S68.

32. Tousignant, M., Boissy, P., Corriveau, H., \& Moffet, H. (2007). In home telerehabilitation for older adults after discharge from acute hospital or rehabilitation unit : A proof of concept study and costs estimation. Disability and Rehabilitation: Assistive Technology, 1, 209-216.

33. Wilson, L., Lincoln, M., \& Onslow, M. (2002). Availability, access, and quality of care: Inequities in rural speech pathology services for children and a model for redress. Advances in Speech-Language Pathology, 4(1), 9-22.

34. Yip, M. P., Chang, A. M., Chan, J., \& MacKenzie, A. E. (2003). Development of the Telemedicine Satisfaction Questionnaire to evaluate patient satisfaction with telemedicine: A preliminary study. Journal of Telemedicine and Telecare, 9, 46-50. 